Nota científica

\title{
Un caso de leucismo en larvas de Phyllomedusa bicolor (Anura: Hylidae) reproducidas en cautiverio
}

\author{
[A case of leucism in larvae of Phyllomedusa bicolor (Anura: Hylidae) bred in \\ captivity]
}

\author{
Adán Villagarcia-Olmeño ${ }^{1 *}$ y Enrique Cuevas ${ }^{2}$
}

${ }^{1}$ Universidad Científica del Perú, Av. Abelardo Quiñones km 2.5, Iquitos, Perú

${ }^{2}$ Consultor Privado

*e-mail: adankr@gmail.com

\begin{abstract}
Resumen
En este artículo se reporta el primer caso de leucismo en Phyllomedusa bicolor (Boaddaert 1772). La larva leucística descrita fue resultado de un trabajo de manejo reproductivo en cautiverio, y fue fotografiada 97 días después de su eclosión, mostrando una coloración blanca uniforme a excepción de los reflejos dorados de los flancos y el tono oscuro de los ojos.
\end{abstract}

Palabras clave: Phyllomedusa bicolor, amelanismo, mutación, melanóforos.

Abstract

In this paper we reported the first case of Leucism in one larval specimen of Phyllomedusa bicolor (Boaddaert 1772). The leucistic larva reported was photographed 97 days after hatching, where shows a uniform white color except the golden hues of the flanks and the dark tone of the eyes.

Keywords: Phyllomedusa bicolor, amelanism, mutation, melanophores.

\section{INTRODUCCIÓN}

Las variaciones cromáticas han sido ampliamente documentadas en diversas especies de anfibios, incluyendo el leucismo, mutación que se manifiesta por la ausencia total o parcial de pigmentación debido al déficit de melanina presente en los melanóforos (Brame, 1962; Dyrkacz, 1981; Bechtel, 1995), lo que le confiere un color claro a la piel, blanco o amarillo dorado, excepto en los ojos, que muestran la pigmentación oscura habitual. Este último rasgo permite diferenciarlo del albinismo, ya que los ojos de los anfibios albinos carecen por completo de pigmentación y muestran una coloración rojiza debido a la elevada capilaridad sanguínea de los globos oculares (Dyrkacz, 1981; Mitchell y Mazur, 1998).

Los melanóforos de los anfibios leucísticos no son funcionales, excepto los ubicados en los ojos. Sin embargo, otros cromatóforos como los xántoforos e iridóforos siguen operativos,

Recibido: 01 de octubre de 2014

Aceptado para publicación: 05 de noviembre de 2014 por lo que algunos ejemplares muestran reflejos dorados (Bechtel, 1995).

A nivel mundial, se han citado ejemplares leucísticos en numerosas especies de anfibios, principalmente de urodelos, como en Plethodon cinereus (Mitchell y Mazur, 1998), Ambystoma opacum (Mitchell y Church, 2002), Salamandra salamandra (Arribas y Rivera, 1992). Respecto a los anuros, también se han reportado casos de amelanismo en varias especies, como un ejemplar leucístico de Alytes dickhilleni encontrado en estado larvario y metamorfoseado en cautividad (Benavides et al., 2000), 48 larvas amelanísticas de Pelodytes punctatus (Rivera et al., 2001); indeterminado número de larvas albinas de Rana temporaria (Guyétant, 1967). En cuanto a los anuros arborícolas nocturnos, los reportes en la naturaleza son escasos y se restringen a las formas larvarias, como varios ejemplares albinos de Hyla arborea descritos por Hensley (1959), ya que la piel blanca de los ejemplares metamorfoseados refleja en 
exceso la luz de la luna, exponiéndolos al acecho sistemático de los potenciales depredadores, por lo que sus probabilidades de alcanzar la madurez sexual en la naturaleza y transmitir su gen recesivo del leucismo o albinismo son muy limitadas (Rosenkilde, 1985 \& Betchel, 1995).

Phyllomedusa bicolor es un anuro nocturno y arborícola de gran tamaño, superando algunas hembras los trece centímetros hocico-cloaca (Caramaschi y Cruz 2002), lo que la convierte en la mayor especie del género Phyllomedusa (Wagler 1830); su distribución abarca la cuenca baja amazónica, incluyendo Brasil, Colombia, Perú, Venezuela y las Guyanas (Frost, 2014). Suele habitar en el dosel de los bosques tropicales húmedos, tanto intervenidos como primarios, desplazándose por las ramas con movimientos lentos y pausados (Caramaschi y Cruz, 2002), y tan sólo desciende a niveles inferiores de la vegetación para reproducirse conforme al modelo reproductivo número 24 descrito para anuros por Haddad y Prado (2005).

\section{MATERIALES $\mathbf{Y}$ MÉTODOS}

En el mes de septiembre del 2014, como parte de un trabajo de reproducción en cautiverio de Phyllomedusa bicolor implementado en un fundo privado, se pudo observar la presencia de un renacuajo con coloración aberrante que exhibía un llamativo tono blancuzco. Dicho fundo está ubicado en el asentamiento humano Unión Progreso, comunidad de Peña Negra, km 10 Carretera Iquitos-Nauta en Loreto, Perú.

El espécimen en cuestión provenía de una puesta obtenida a partir de un grupo reproductor compuesto por 12 machos y 2 hembras adultas; una vez localizada la puesta, procedimos a extraerla e incubarla basándonos en los protocolos de manejo reproductivo en cautividad desarrollados por Fenolio (1996).

\section{RESULTADOS Y DISCUSIÓN}

La puesta en cuestión eclosionó al cabo de diez días de incubación, dando como resultado 585 larvas viables. Posteriormente 486 renacuajos completaron su desarrollo entre 46 a 94 días, con un promedio de 59 días.

La coloración de casi todos los renacuajos de Phyllomedusa bicolor fue homogénea, excepto por el ejemplar blanco fotografiado (Figura 1). Tras haber obtenido cuatro puestas eclosionadas con éxito y más de 3000 renacuajos de esta especie, pertenecientes a tres hembras distintas y 24 potenciales machos, tan sólo hemos observado este espécimen con un morpho aberrante.

El resto de las larvas mostraron una coloración parduzca (Figura 2) sin cambios aparentes hasta dos o tres días después de completar su metamorfosis, momento en el cual cambian progresivamente del verde oscuro mate a un verde brillante y lustroso.

Sin embargo, el ejemplar que se reporta muestra una coloración blanca uniforme, a excepción de los reflejos dorados (xántoforos e iridóforos operativos) de los flancos y el tono oscuro (melanóforos funcionales) de los ojos, rasgo determinante en la caracterización del leucismo (Bagnara et al., 1979; Betchel, 1995; Burgess, 1994).

Para el caso de la especie Phyllomedusa bicolor no existen reportes previos de amelanismo. En el presente trabajo se reporta el primer caso de leucismo para este anuro.

Dado que los parámetros físico-químicos del agua y la dosificación y frecuencia de administración del alimento fueron idénticos para todas las puestas eclosionadas, descartamos estos factores como causa del carácter leucístico. Teniendo en cuenta que el leucismo es un carácter genético recesivo, si una cría muestra externamente su coloración aberrante su genotipo debe ser $(\mathrm{mm})$, y para ello sus dos progenitores de coloración verde estándar deberían ser portadores heterocigotos del gen recesivo del Leucismo (Mm).

Pero en tal caso, el $25 \%$ del total de las larvas deberían mostrar un morpho aberrante, y sin embargo el porcentaje resultante no alcanza el 0,5\% del total de los renacuajos de esa puesta. Así mismo, la manifestación física externa de la mutación leucística le supone un hándicap para su supervivencia en la naturaleza, al reducir significativamente sus probabilidades de alcanzar la madurez sexual y poder transmitir sus caracteres leucísticos a la siguiente generación. Por ello, consideramos la posibilidad de que este singular caso sea causado por una mutación espontánea. 


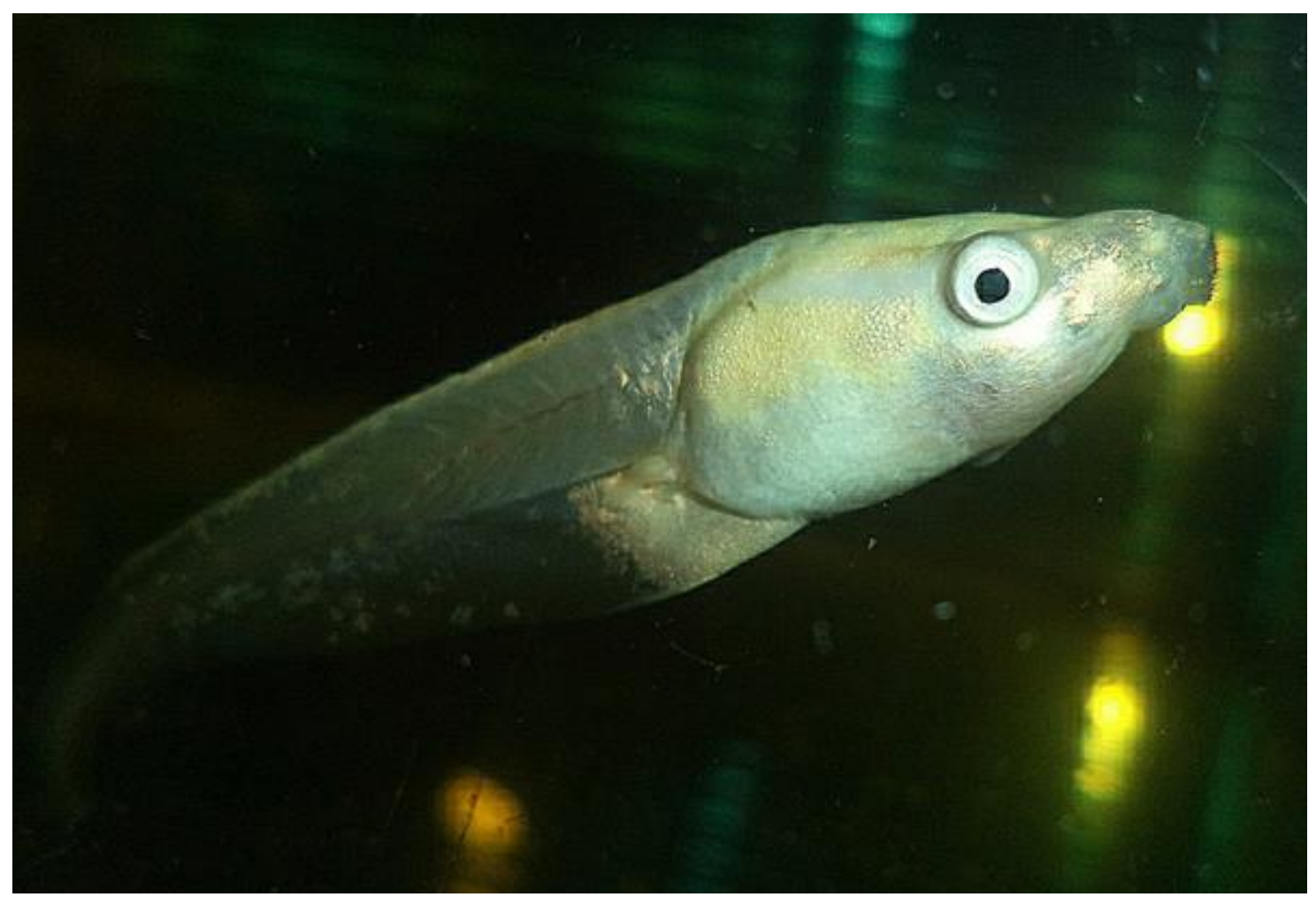

Figura 1. Larva leucística de Phyllomedua bicolor

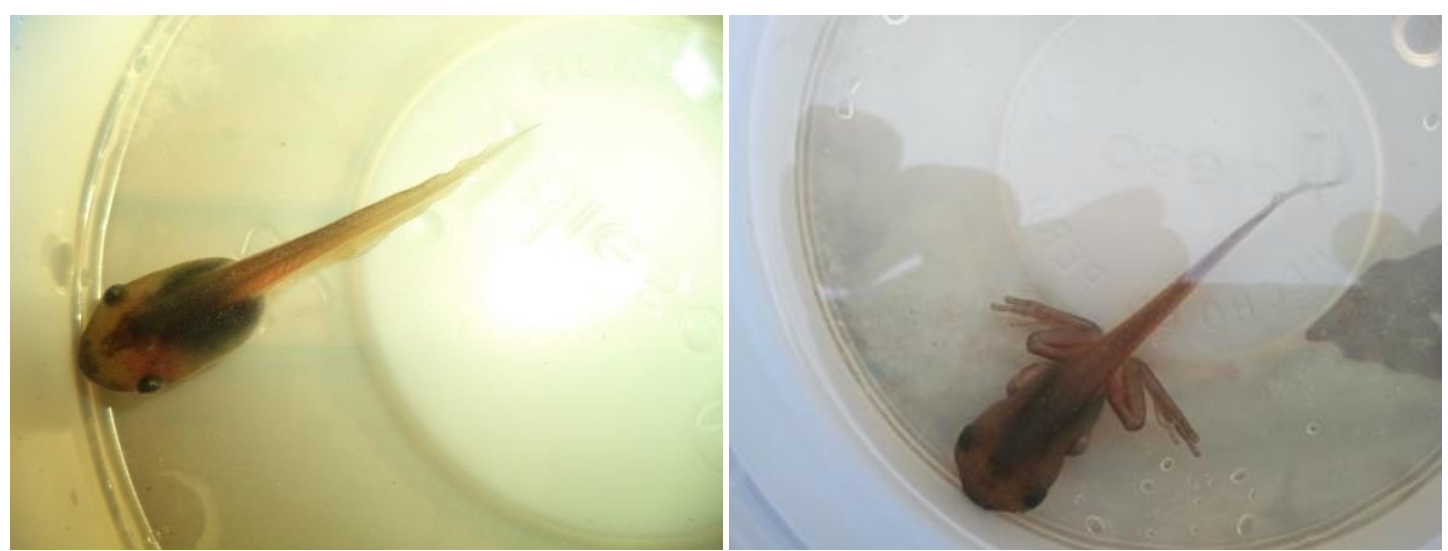

Figura 2. Larvas de Phyllomedusa bicolor morpho "estándar" 


\section{CONCLUSIONES}

El reporte de este primer caso de leucismo para Phyllomedusa bicolor en especímenes larvarios reproducidos en cautividad resulta relevante dado el reducido número de casos descritos para anuros arborícolas nocturnos.

\section{REFERENCIAS BIBLIOGRÁFICAS}

Arribas O, Rivera J. 1992. Albinismo en Salamandra salamandra (Linnaeus, 1758) en el noreste ibérico. Boletín Asociación Herpetológica Española 3: 1415.

Bagnara JT, Frost SK, Matsumoto J. 1979. On the development pigment patterns in Amphibians. Amer Zool 18: 301-312.

Benavides, J., Viedma, A., Clivilles, J., Ortiz, A. \& Gutiérrez, J.M. 2000. Albinismo en Alytes dickhilleni y Salamandra salamandra en la Sierra de Castril (Granada). Boletín de la Asociación Herpetológica Española 11: 83.

Betchel HB. 1995. Reptile and Amphibian variants. Color, Patterns, and sxales. Krieger Publishing Company Malabar, Florida. 206 pp

Brame AH Jr. 1962. A survey of albinism in salamanders. Abhandlungen und Berichte Naturkunde Vorgeschichte 11: 65-81.

Burgess T. 1994. What is Albino? A glossary of terms and a description of some available colour mutations in reptiles and amphibians. Reptilian 4(2): 6-8.

Caramaschi U, Cruz CAG. 2002. Phyllomedusa: taxonomía, hábitos y biología (Amphibia, Anura, Hylidae). Phyllomedusa 1: 5-10.

Dyrkacz S. 1981. Recent instances of albinism in North American amphibians and reptiles. Society for the Study of Amphibians and Reptiles. Herpetological Circular 11, 31 pp.
Fenolio D. 1996. Captive Reproduction of the Orange-legged Monkey Frog (Phyllomedusa hypocondrialis), Development of a Protocol for Phyllomedusine Frog Reproduction in the laboratory; Advances in Herpetoculture, pages 13-21.

Frost DR. 2014. Amphibian species of the world: an online reference. Version 6.0 (Date of Access). Electronic Database accesible. American Museum of Natural History, New York, USA. http://research.amnh.org/herpetology/amp hibia/index.html

Guyétant R. 1967. Les groupements de reproduction chez quelques amphibiens anoures et leurs consequences sur la vie larvaire. Vie et Milieu, 26: 91-114.

Haddad CFB, Prado CPA. 2005. Reproductive modes in frogs and their unexpected diversity in the Atlantic Forest of Brazil. Bioscience 55:207-217.

Hensley M. 1959. Albinism in North American amphians and reptiles. Publication of the Museum, Michigan State University 1: 133-159.

Mitchell JC, Mazur J. 1998. Leucistic RedBaced Salamanders (Plethodon cinereus) from Maryland. Northeastern Naturalist 5(4): 367-369.

Mitchell JC, Church DR. 2002. Leucistic Marbled Salamanders (Ambystoma opacum) in Virginia. Banisteria 20: 6769.

Rivera X, ARRIBAS O, MARTI F. 2001. Anomalías pigmentarias en anfibios y reptiles. Quercus 180: 18.22. 